\title{
Smart Contrast Agents for Magnetic Resonance Imaging
}

\author{
Célia S. Bonnet and Éva Tóth*
}

\begin{abstract}
By visualizing bioactive molecules or biological parameters in vivo, molecular imaging is searching for information at the molecular level in living organisms. In addition to contributing to earlier and more personalized diagnosis in medicine, it also helps understand and rationalize the molecular factors underlying physiological and pathological processes. In magnetic resonance imaging (MRI), complexes of paramagnetic metal ions, mostly lanthanides, are commonly used to enhance the intrinsic image contrast. They rely either on the relaxation effect of these metal chelates ( $T_{1}$ agents), or on the phenomenon of paramagnetic chemical exchange saturation transfer (PARACEST agents). In both cases, responsive molecular magnetic resonance imaging probes can be designed to report on various biomarkers of biological interest. In this context, we review recent work in the literature and from our group on responsive $T_{1}$ and PARACEST MRI agents for the detection of biogenic metal ions (such as calcium or zinc), enzymatic activities, or neurotransmitter release. These examples illustrate the general strategies that can be applied to create molecular imaging agents with an MRI detectable response to biologically relevant parameters.
\end{abstract}

Keywords: Lanthanide complexes · Magnetic resonance imaging · Molecular imaging · Paramagnetic complexes · Responsive probes

\section{Introduction}

Traditionally, magnetic resonance imaging (MRI) has long been used to obtain anatomical and functional images. In recent years, the new field of molecular imaging has emerged which looks for information at the molecular level by visualizing the concentration and function of bioactive molecules or physiological parameters. By revealing the biochemical or physiological abnormalities as the origin of the diseases, much earlier than the structural consequences of these abnormalities become visible, molecular imaging allows earlier and more personalized diagnosis but also contributes to the understanding and rationalization of the molecular factors underlying pathological processes. Particularly important targets involve $\mathrm{pH}$, temperature, concentration of ions or metabolites. Unlike anatomic imaging, molecular imaging always needs an imaging agent, called

${ }^{*}$ Correspondence: Dr. E. Tóth

Centre de Biophysique Moléculaire

CNRS, UPR4301, Université d'Orléans

Rue Charles Sadron, 45071 Orléans, France

E-mail: eva.jakabtoth@cnrs-orleans.fr smart or responsive, that will be able to provide an MRI detectable response depending on the specific parameter that we want to image. MRI, a non-invasive technique endowed with a high resolution, is particularly attractive for the development of such applications. Traditionally, MRI probes can be divided in two categories: $T_{1}$ and $T_{2}$ contrast agents that respectively modify the longitudinal and transverse relaxation time of the surrounding water molecules. $T_{1}$ agents are mainly $\mathrm{Gd}^{3+}$ chelates, and to a lesser extent, $\mathrm{Mn}^{2+}$ chelates, and $T_{2}$ agents are iron oxide nanoparticles. ${ }^{[1]}$ Since the early 2000s, chemical exchange saturation transfer (CEST) probes have emerged as a new class of potential paramagnetic MRI contrast agents. PARACEST (paramagnetic CEST) agents are paramagnetic complexes either of lanthanide (except gadolinium), or of transition metal ions which possess paramagnetically shifted exchangeable protons. ${ }^{[2]}$ In this short review, we will focus on $\mathrm{Ln}^{3+}$ chelates for MRI, i.e. $\mathrm{Gd}^{3+}$ based contrast agents and PARACEST agents.

The efficacy of $\mathrm{Gd}^{3+}$-based probes to accelerate water proton relaxation is called relaxivity (paramagnetic relaxation enhancement of water proton relaxation rates per millimolar concentration of $\mathrm{Gd}^{3+}$ ). The relaxation behavior and the underlying mechanisms have been discussed elsewhere. ${ }^{[1,3]}$ The relaxivity is inherently linked to the microscopic parameters of the $\mathrm{Gd}^{3+}$ complex. The most important microscopic factors are i) the number of water molecules directly coordinated to the metal ion (hydration number, $q$ ), ii) the exchange rate, $k_{\mathrm{ex}}$, of these water molecules with the surrounding water and iii) the rotational correlation time, $\tau_{R}$, of the agent (Fig. 1a). To design a smart contrast agent, the relaxivity has to be selectively influenced by the biomarker that we want to detect. Any of these microscopic parameters that determine relaxivity can be modulated by the presence of a specific biomarker. In practice, smart contrast agents mostly function based on changes in the hydration number or in the rotational correlation time of the complex. ${ }^{[4]}$ The reason is that these parameters are the most amenable to chemical modulation and the easiest to predict.

For PARACEST agents, the exchangeable protons of the paramagnetic complexes (-NH of amides, amines or-OH of water or alcohols) are in slow exchange with bulk water protons (Fig. 1b). ${ }^{[2]}$ Radiofrequency pulses applied at the appropriate frequency are used to selectively saturate these protons, and the chemical exchange between these and the bulk water protons will lead to a diminution of the water proton signal intensity which will be translated to a MR image. PARACEST smart probes can be developed by modulating the concentration, the exchange rate or the resonance frequency of those mobile protons by the presence of the biomarker.

Responsive probes, like any metalbased imaging agent, are required to possess high thermodynamic stability and kinetic inertness in order to avoid toxicity related to the release of free $\mathrm{Ln}^{3+}$ ions. This toxicity is mainly related to the simi- 


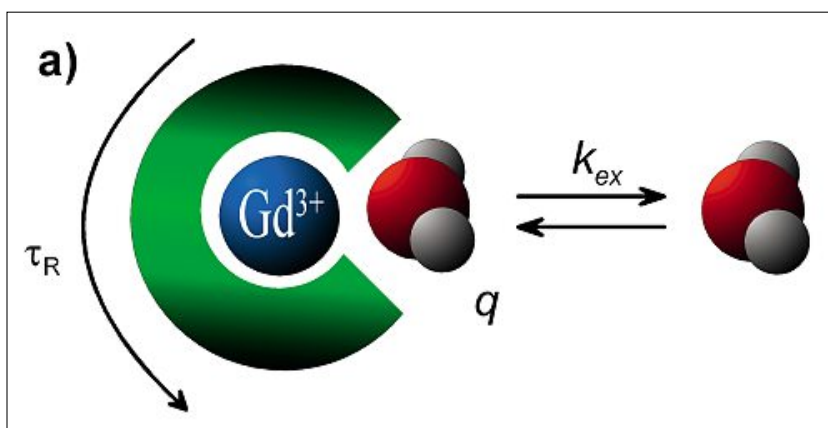

b)

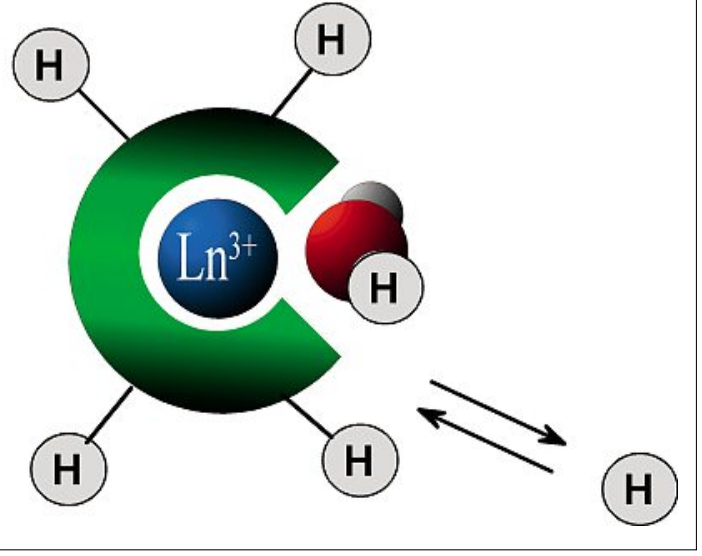

lar size of $\mathrm{Ln}^{3+}$ compared to $\mathrm{Ca}^{2+}$, that they can replace in vivo, and also to the possible formation of hydroxo complexes around physiological $\mathrm{pH}$. Consequently, most of the molecular imaging probes reported in the literature are based on DTPA- or DOTA-type chelating units which ensure sufficient thermodynamic and kinetic stability. When modulating the ligand structure, in particular by changing the hydration number, attention has to be always paid to maintain high kinetic and thermodynamic stability of the resulting complexes.

In this review, we will discuss recent efforts made by us and others in the field of responsive probes, more particularly for cation detection (calcium and zinc), enzyme, and neurotransmitter sensing. The aim is not to be exhaustive, but to illustrate the strategy used, the advantages and the limitations on several examples.

\section{Detection of Enzymatic Activities}

Enzymes are important biomarkers to detect in vivo since they have a central role in biological systems both in the normal physiological state and various pathologies. MRI is often considered as adapted to enzymatic detection despite its low sensitivity. The reason is that even at very low concentrations, an enzyme can catalytically convert a high amount of the smart imaging agent, making it perfectly detect-
Fig. 1. a) Major parameters influencing relaxivity, thus $\mathrm{MRI}$ efficiency of a $\mathrm{Gd}^{3+}$ complex: the hydration number (water molecules directly coordinated to the $\left.\mathrm{Gd}^{3+}\right), q$, the water exchange rate between the inner coordination sphere and the bulk, $k_{\text {ex }}$, and the rotational dynamics of the chelate, characterized by the rotational correlation time, $\tau_{R}$. b) Paramagnetic chemical exchange saturation transfer (PARACEST) agents: the PARACEST effect results from proton exchange between the bulk water and protons on the ligand or on the coordinated water molecules of the lanthanide chelate.

able. Therefore the literature on MRI detection of enzymes has increased exponentially since the first report of Meade and co-workers on $\beta$-galactosidase detection at the end of the 1990s. ${ }^{[5]}$ Oxydoreductases, transferases, and hydrolases have been investigated, and both PARACEST agents and $\mathrm{Gd}^{3+}$ complexes have been proposed for their detection. ${ }^{6]}$

First, for $\mathrm{Gd}^{3+}$ chelates, the change of relaxivity can be due to a change in the hydration number of $\mathrm{Gd}^{3+}, q$. Historically, the first enzymatically responsive contrast agent, developed by Meade et al., was based on this concept. ${ }^{[5]}$ Indeed, the GdDO3A derivative Gd1 (Fig. 2) bears a galactopyranose residue which sterically prevents water coordination to the $\mathrm{Gd}^{3+}$ ion. In the presence of $\beta$-galactosidase, the sugar moiety is cleaved from the complex, opening the access for water molecules to $\mathrm{Gd}^{3+}$. This molecule could be applied with success to image $\beta$-galactosidase messenger ribonucleic acid expression in living Xenopus laevis embryos. ${ }^{[7]}$ The use of a self-immolative arm has also been proposed, i.e. after enzymatic cleavage an electronic cascade gives rise to a contrast agent with an additional coordinating group, leading to a decrease of the relaxivity. ${ }^{[8]}$

Rotational correlation time $\left(\tau_{\mathrm{R}}\right)$ changes have also been exploited. As $\tau_{R}$ is linked to the size and flexibility of the complex, this has been particularly used in the case of oxydoreductases and transferases. Indeed, these enzymes activate the probe by catalyzing the transfer of electrons and chemical bond formations leading to oligomerization for the former, and by cross-linking between proteins to form aggregates through intermolecular lysine bond formation for the latter. In both cases, this leads to higher molecular weight complexes, with a longer rotational correlation time (higher $\tau_{R}$ ), thus a higher relaxivity. GdDTPA-diTyr Gd2 (Fig. 2) bearing tyramide and hydroxytryptamide moieties was proposed for peroxidase and tyrosinase imaging. ${ }^{[9]}$ Following the enzymatic reaction, a net increase in longitudinal relaxivity explained by a slower rotation of the oligomer compared to the monomer was observed. When the hydroxytyramide was substituted by a 5-hydroxytryptamide (serotonin) unit, the myeloperoxidase (MPO) enzyme, secreted by leukocytes during inflammation, could be imaged.[10]

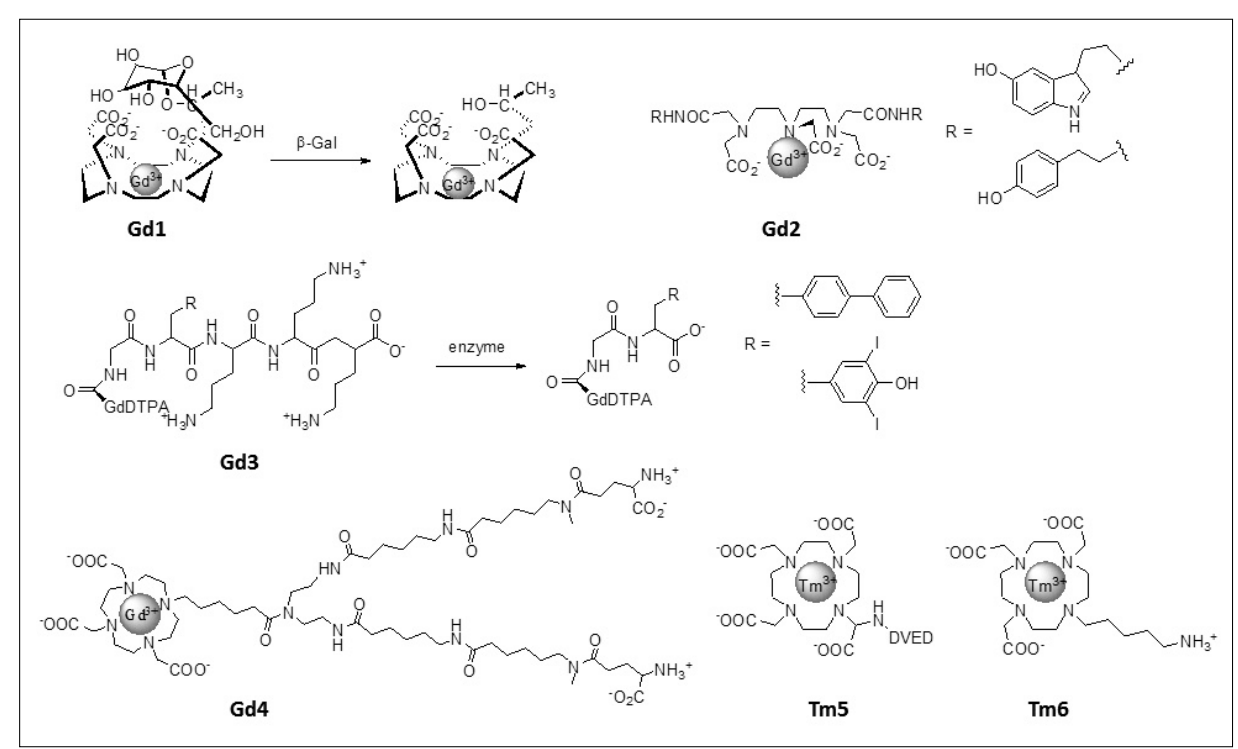

Fig. 2. $\mathrm{Ln}^{3+}$ complexes for enzymatic detection. 
Even if the modulation of rotational dynamics is more common for the detection of enzymes generating oligomers, it has also been elegantly used for hydrolases through exploiting the difference of affinity of the imaging probe for HSA (human serum albumin) before and after enzymatic cleavage.[11] Gd3 (Fig. 2) can be cleaved by human carboxypeptidase $\mathrm{B}$, an enzyme that has a role in thrombotic disease. This imaging probe is built up by combining four moieties: a masking group (three lysine residues), an HSA binding group, a glycine linker, and the paramagnetic MRI reporter GdDTPA. While the complex is a poor albumin binder before enzymatic reaction, after enzymatic cleavage, its affinity to serum albumin is increased which results in a higher relaxivity.

Changes in both $q$ and $\tau_{\mathrm{R}}$ can be also exploited together for a maximum difference in relaxivities. This is the case of the GdDO3A derivative Gd4 (Fig. 2) which contains two glutamate moieties, and which is responsive to the glutamic acid decarboxylase, present in neurons. ${ }^{[12]} \mathrm{Up}$ on enzymatic reaction, $q$ increases and the positive charge created enhances the binding of the agent to macromolecules, thus increasing $\tau_{R}$.

Solubility differences have also been used to detect enzymatic activity. Matrix metalloproteinase-2 (MMP-2) plays a role in tumor development.[13] The agent has three different parts: i) a MMP-2 cleavable peptide substrate, ii) a GdDOTAMA chelate conjugated to a hydrophobic chain, and iii) a polyethylene glycol chain (PEG). Upon cleavage of the substrate by the MMP-2 enzyme, the PEG chain is removed from the $\mathrm{Gd}^{3+}$ chelate, thus its aqueous solubility is considerably reduced, leading to a relaxivity decrease. The agent could be used to image different levels of enzyme activity in a tumor-bearing animal model.

Caspase has been detected by ${ }^{19} \mathrm{~F}$ MRI with a probe that contained a peptide sequence substrate of Caspase- 3 conjugated on one side to a $\mathrm{Gd}^{3+}$ chelate and on the other to a ${ }^{19} \mathrm{~F}$-containing moiety. ${ }^{[14]}$ Before enzymatic cleavage, the $T_{2}$ of the ${ }^{19} \mathrm{~F}$ signal is quenched by the paramagnetic $\mathrm{Gd}^{3+}$ (short $T_{2}$, broad peak), whereas after, the $\mathrm{Gd}^{3+}$ - and the ${ }^{19} \mathrm{~F}$-containing moieties are separated, making the ${ }^{19} \mathrm{~F}$ signal observable.

Finally, PARACEST agents represent a good strategy to detect enzymatic activities as many enzyme substrates contain hydroxyl, amine and amide groups with labile protons that can be readily detectable by CEST. The first example contains a TmDOTA unit linked to DEDV, the peptide substrate of Caspase-3 (Tm5, Fig. 2). ${ }^{[15]}$ Upon enzymatic reaction, the amide protons disappear, switching off the related CEST effect. A similar PARACEST agent has been used with a different peptide for monitoring the activity of urokinase plasminogen activator.[16] The main drawback of these systems is a 'turn-off' response of the CEST effect upon enzymatic activity. Recently a 'turn-on' system for the detection of transglutaminase has been proposed.[17] A Tm-DO3A-cadaverine Tm6 creates a covalent bond with albumin upon enzymatic activation, and the amide created generates a CEST effect.

The concept of a self-immolative linker has also been used for PARACEST compounds. Indeed, we have developed an $\mathrm{Yb}$ complex, Yb7, linked to a $\beta$-D-galactopyranoside substrate via a self-immolative benzyloxycarbamate for $\beta$-galactosidase detection (Fig. 3). The enzymatic removal of the substrate leads to a electron cascade reaction and consequently yields the spontaneous removal of the linker. A PARACEST signal appears for the final product, which could be related to slowly exchanging amine protons. This is surprising since amine protons usually undergo fast exchange, which would not lead to an observable PARACEST effect. In this coordination compound, however, proton exchange is much slower, due to the binding of the adjacent carboxylate and the macrocyclic amine to the metal cation which brings about unusual properties of the exocyclic $\mathrm{NH}_{2}$. The protonation constant of this amine, as determined by $\mathrm{pH}$-potentiometry for $\mathrm{Gd}\left(\mathrm{DOTA}-\mathrm{NH}_{2}\right)$, is remarkably low $\left(\log K_{\mathrm{H}}=5.12\right)$ compared to those for typical amines. This implies that the amine in $\mathrm{Yb}\left(\mathrm{DOTA}-\mathrm{NH}_{2}\right)$ is unprotonated at and above physiological $\mathrm{pH}$ and this is certainly the reason for the slow proton exchange. ${ }^{[18]}$ The advantage of such a design is that the system functions as an 'OFF-ON' probe and that by simply changing the substrate, a whole range of enzymes can be detected, with a
PARACEST effect that can be optimized once for all enzymes.

\section{Detection of Endogenous Cations}

The detection of endogenous cations by imaging approaches is becoming increasingly important. Their concentration is strictly regulated in biological systems. Any disturbance in their homeostasis is linked to pathological states involving cancer or neurodegenerative diseases. Imaging can be interesting to achieve in vivo quantification of these ions in living systems in order to better understand their role and to relate abnormalities to pathologies thus developing novel approaches for early diagnosis. Optical imaging has been long used for their detection and clearly contributed to a better understanding of their homeostasis. However, optical imaging is intrinsically limited to superficial tissues. MRI can therefore offer a good alternative. The responsive probes developed for cation detection are generally composed of two moieties: the MRI-reporter unit $\left(\mathrm{Ln}^{3+}\right.$ chelate) and a binding site specifically designed to chelate the ion to detect. As for enzymatic detection, the two main approaches to lead to a relaxivity change of $\mathrm{Gd}^{3+}$-based agents through metal ion binding rely on a modulation of the hydration number, $q$, or of the rotational correlation time, $\tau_{R}$ as illustrated in Fig. 4. In the first strategy, one (or several) donor atoms belonging to the metal specific binding unit are weakly bound to the $\mathrm{Gd}^{3+}$ in the absence of the sensed ion. When the sensed cation is present and enters the coordination pocket, these donor groups will flip from the $\mathrm{Gd}^{3+}$ and coordinate the other ion, and this will allow hydration water molecules to enter the coordination sphere

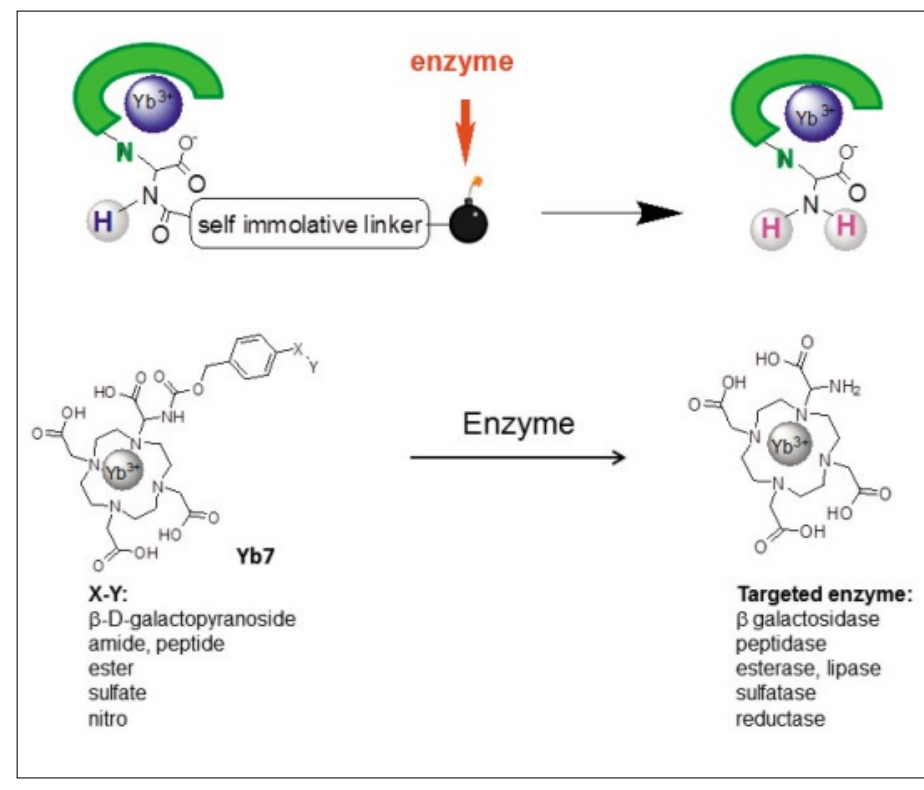

Fig. 3. Schematic and molecular representation of the self-immolative concept for enzyme detection. 
of $\mathrm{Gd}^{3+}$, yielding an increase in relaxivity (Fig. 4a). The second approach takes advantage of the formation of supramolecular assemblies upon metal ion coordination to the imaging probe, resulting in larger molecular weight, longer rotational correlation times $\tau_{R}$ and consequently higher relaxivities than the starting mononuclear complex (Fig. 4b). For the development of metal-responsive agents, one important issue is the design of the specific metal binding site. It has to be selective, provide reversible binding and should be adapted to the physiological concentration range.

\subsection{Calcium Sensing}

The physiological role of calcium is related to its importance in signal transduction pathways, in neurotransmitter release from neurons, contraction of all muscle cell types, and fertilization. Extracellular calcium is also responsible for ensuring the potential difference across cell membranes. The majority (99\%) of the total calcium body content is in bones and calcified cartilage. Calcium is an important constituent of the extracellular and intracellular fluids, with three orders of magnitude higher concentration in the extracellular than in the intracellular space.

Several $\mathrm{Ca}^{2+}$ responsive contrast agents are based on two DO3A derivatives for $\mathrm{Gd}^{3+}$ complexation bridged by a $\mathrm{Ca}^{2+}$ specific binding site. ${ }^{[19]}$ The first agent reported was GdDOPTA Gd8 (Fig. 5), where the BAPTA ${ }^{4-}$ unit is used for $\mathrm{Ca}^{2+}$ complexation. ${ }^{[20]} \mathrm{BAPTA}^{4-}$ is a $\mathrm{Ca}^{2+}$ selective fluorescent chelator which was proposed earlier by Tsien. ${ }^{[21]}$ Among its advantages, one can cite good selectivity for $\mathrm{Ca}^{2+}$ over other endogenous cations and $\mathrm{pH}$ insensitivity of $\mathrm{Ca}^{2+}$ complexation. GdDOPTA shows a $77 \%$ relaxivity increase when it binds $\mathrm{Ca}^{2+}$. Its dissociation constant is in the micromolar range corresponding to intracellular concentrations. The increase of the number of water molecules coordinated to $\mathrm{Gd}^{3+}$ has been proven to be responsible for the relaxivity increase observed. When MRI detection is concerned, targeting the extracellular $\mathrm{Ca}^{2+}$ concentration range (millimolar) is more adapted, as these higher concentrations are more accessible for an MRI probe. In addition, it also simplifies the design of the probe as no cell internalization is required. Consequently, we proposed to replace two carboxylate functions of the BAPTA by less coordinating amide functions to decrease the affinity for $\mathrm{Ca}^{2+}{ }^{2}{ }^{22]}$ The BAPTA was also replaced by bisamide derivatives of EDTA, DTPA $^{[23]}$ or EGTA ${ }^{[24]}$ (Gd9, Fig. 5). In this last example, the relaxivity increase, of $c a$. $70-80 \%$ of Gd9, could be related to the increase in the hydration number $q$, and to the slight rigidification of the complex following the binding of $\mathrm{Ca}^{2+}$ in the central

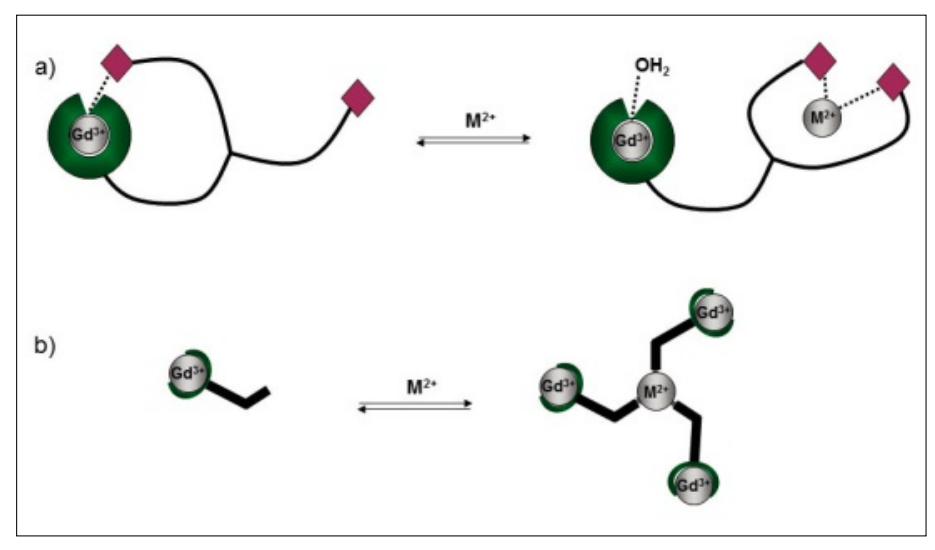

Fig. 4. Two approaches to modulate relaxivity for cation sensing: a) through $q$ modulation, b) through $\tau_{R}$ modulation. part of the molecule. The relaxivity changes are also retained in solutions mimicking the brain extracellular medium, or an in vitro cell culture with astrocytes).[24,25] More recently, these systems were also derivatized to obtain a dual frequency ${ }^{1} \mathrm{H},{ }^{19} \mathrm{~F}$ MRI reporter, ${ }^{[26]}$ or to obtain a dendrimeric contrast agent which displays optimized diffusion properties compared to the monomeric form. ${ }^{[27]}$

We have also developed a PARACEST contrast agent based on a DOTAM derivative to which four iminodiacetate groups have been appended for $\mathrm{Ca}^{2+}$ binding.[28] A CEST effect is observed for both $\mathbf{Y b 1 0}$ and Eu10 complexes and it originates respectively from the exchangeable protons of the amide functions, and the coordinated water. The CEST effect decreases considerably upon $\mathrm{Ca}^{2+}$ and $\mathrm{Mg}^{2+}$ binding. The affinities observed are low, suggesting the coordination of one iminodiacetate arm. We proved that $\mathrm{Ca}^{2+}$ and $\mathrm{Mg}^{2+}$ coordination to the Yb10 complex decreases the exchange rate of the amide protons. Phantom MRI images in the presence of absence of $\mathrm{Ca}^{2+}$ were obtained at $16 \mathrm{~T}$.

\subsection{Zinc Sensing}

Zinc detection has also attracted interest in the past few years as zinc is the second most abundant transition metal in the body after iron, and it is highly present in the brain (up to $300 \mu \mathrm{M}$ in vesicles of certain glutamatergic neuronal cells). ${ }^{[29]}$ Its misregulation can imply excitotoxic neuronal death, particularly during epileptic seizure or head trauma, and it has also been connected to neurodegenerative diseases such as Alzheimer's or Parkinson's.

Ligand design for the detection of zinc is mainly based on a DTPA or a DOTA unit to which a zinc-binding moiety has been appended, involving either iminodiacetate functions ${ }^{[30]}$ or, more often, a DPA (bis-(2-pyridylmethyl)amine) unit.[19] Indeed, DPA possesses good affinity $\left(K_{\mathrm{d}}\right.$ $\approx 25 \mathrm{nM}$ ), and good selectivity for $\mathrm{Zn}^{2+}$. DPA has been further modified for $\mathrm{Zn}^{2+}$ sensing, and the highest relaxivity changes have been reported for a DPA derivative in which one pyridine has been replaced by a carboxylate group. ${ }^{[31]}$ For the iminodiacetate, in the case of Gd12 (Fig. 6), the selectivity for $\mathrm{Zn}^{2+}$ over $\mathrm{Cu}^{2+}$ is not good. [32] No in vivo imaging has been obtained with these probes but relaxivity measurements of Gd12 in male human blood serum evidenced that changes remained sizeable under physiological conditions (33\% vs $73 \%$ in vitro). ${ }^{[33]}$

The most successful agent so far which proved the in vivo feasibility of cation detection by MRI is a GdDOTA-bisamide complex bearing two DPA moieties (Gd13, Fig. 6). ${ }^{[34]}$ The relaxivity remarkably increases upon $\mathrm{Zn}^{2+}$ binding and in the presence of HSA, due to an increase in the

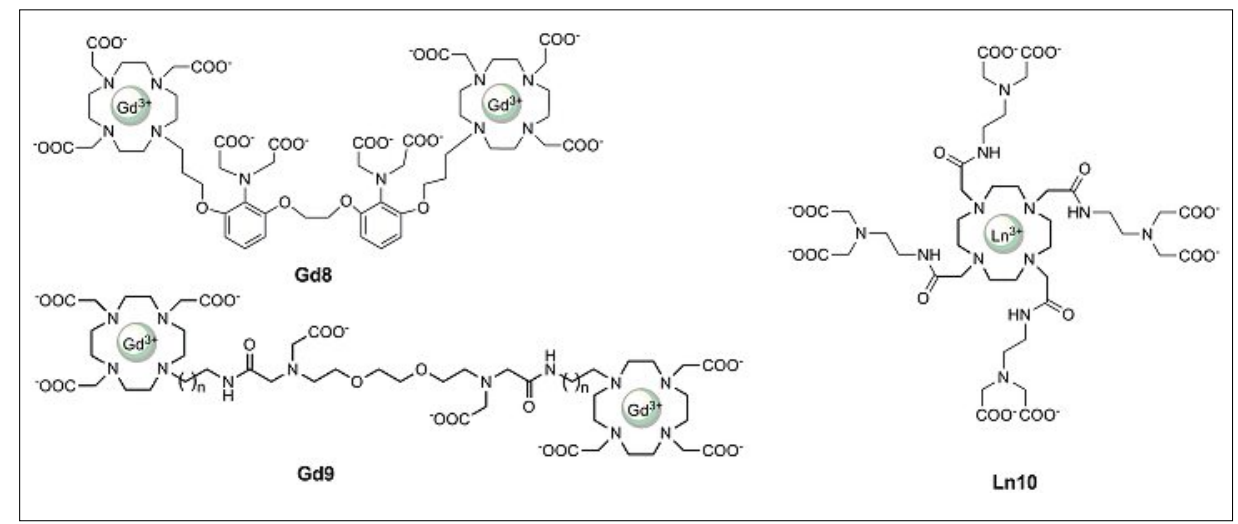

Fig. 5. $\mathrm{Ln}^{3+}$ probes for $\mathrm{Ca}^{2+}$ sensing. 


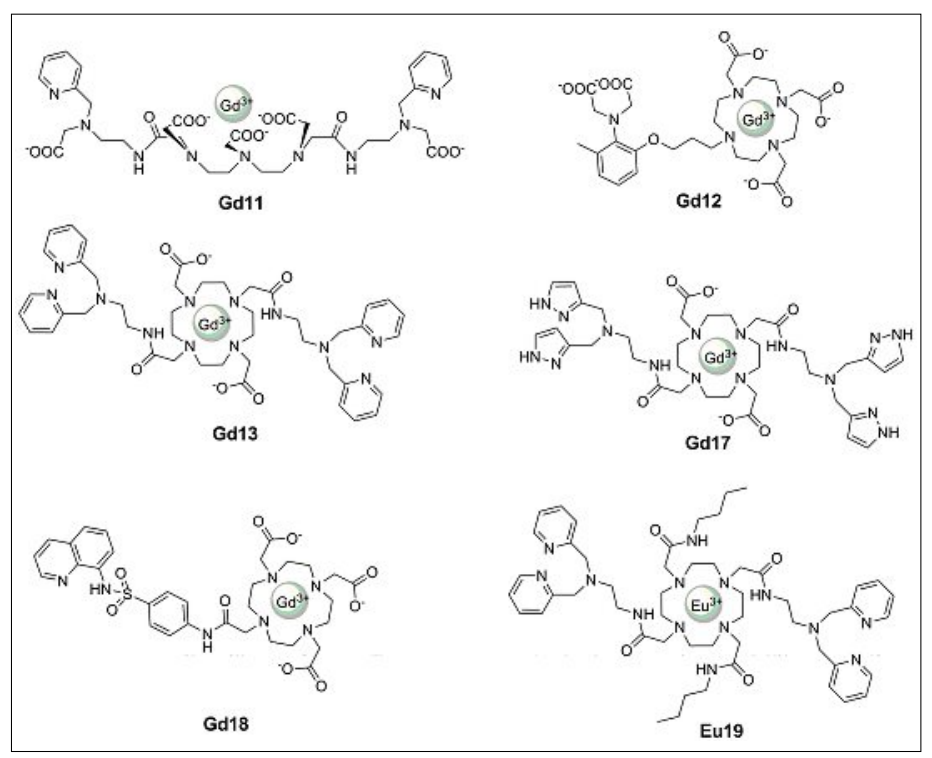

Fig. 6. DOTA and DTPA-based contrast agents for $\mathrm{Zn}^{2+}$ detection.

size of the complex resulting from the remarkable HSA binding affinity of the $\mathrm{Zn}^{2+}$ bound complex. In the absence of $\mathrm{Zn}^{2+}$, the complex hardly interacts with HSA. This complex has been used in vivo for imaging $\beta$-cells in mouse pancreas. Indeed, in response to high glucose levels, $\mathrm{Zn}^{2+}$ is released simultaneously with insulin from $\beta$-cells. ${ }^{[35]}$ Image contrast was significantly enhanced in the mouse pancreas after injection of glucose and Gd13, whereas without glucose and in the presence of Gd13 no enhancement was observed. This nicely demonstrates that $\mathrm{Zn}^{2+}$ sensors can potentially be very useful tools to detect the progression of type 2 diabetes.

Encouraged by these positive results, we have also developed a series of $\mathrm{Gd}^{3+}$ based contrast agents for $\mathrm{Zn}^{2+}$ detection (Gd14-Gd16, Fig. 7), based on a pyridine unit for $\mathrm{Gd}^{3+}$ complexation. [36] We had previously shown that the bishydrated $\mathrm{Gd}^{3+}$ complex possesses optimized MRI properties (particularly water exchange rate), while retaining a good thermodynamic and kinetic stability.[37] The DPA unit has been modified by introducing coordinating carboxylate functions either in the place of one pyridine of the DPA (Gd14), or on the pyridine of the DPA (Gd15, Gd16). Finally the linkers were an alkyl chain, or an alkyl chain of different length bearing an amide function. The aim was to perform a systematic study of the parameters governing the relaxivity of those complexes to guide us towards a rational design of more efficient complexes for zinc sensing. First, we have shown that the presence of the amide function in the linker is necessary for achieving a sufficient stability of the $\mathrm{Gd}^{3+}$ complex with $\mathrm{Zn}^{2+}$. Then, with combined ${ }^{1} \mathrm{H},{ }^{13} \mathrm{C},{ }^{17} \mathrm{O} \mathrm{NMR}$, and proton relaxivity measurements we have unambiguously proved that the $c a .20 \%$ relaxivity changes observed were due to the formation of a dimeric species with a high rotational cor- relation time. We could estimate a dissociation constant for $\mathrm{Zn}^{2+}$ at physiological $\mathrm{pH}$ which is in the relevant nanomolar range. Finally the selectivity for $\mathrm{Zn}^{2+}$ over other physiological cations was also sufficient, validating this $\mathrm{Zn}^{2+}$ complexing unit. We are currently working on new systems with optimized thermodynamic stability and $\mathrm{Zn}^{2+}$ response.

Other $\mathrm{Zn}^{2+}$ complexing units have been tested, such as 3-pyrazolyl[38] (Gd17, Fig. $6)$ and 8-sulfonamidoquinoline ${ }^{[39]}$ (Gd18, Fig. 6). The affinity of the former for $\mathrm{Zn}^{2+}$ is $K_{\mathrm{D}}=380 \mu \mathrm{M}$, much weaker than that of DPA. $\left(K_{\mathrm{D}}=500 \mathrm{nM}\right)$. For the quinoline, the affinity is $K_{\mathrm{D}}=500 \mathrm{nM}$, and as it is also a chromophore, the complex has been proposed as a bimodal responsive probe for both MRI and fluorescent imaging. The para position of the sulfonamide moiety on the benzene allows for the formation of a (Gd18) Zn species only, with an affinity constant in the pM range. ${ }^{[40]}$ This modulation of zinc affinity should allow for the detection of a wide range of $\mathrm{Zn}^{2+}$ concentration, which is crucial to get a clearer picture of zinc homeostasis in vivo. However one should always be careful with high affinity sensors not to disturb the homeostasis.

Finally, it should be noted that $\mathrm{Zn}^{2+}$ binding can also modulate the PARACEST effect. The $\mathrm{Eu}^{3+}$ complex Eu19 is structurally similar to Gd13 but contains two amide functions instead of two carboxylate groups (Fig. 6). ${ }^{[41]}$ The amide arms contribute to slowing down the water exchange rate which makes it possible to benefit from a PARACEST effect originating from the water molecule coordinated to $\mathrm{Eu}^{3+}$. The underlying mechanism leading to the differences in PARACEST has not been fully understood, but it has been proposed that this chelate can detect $\mathrm{Zn}^{2+}$ concentration changes ranging from $5 \mathrm{nM}$ to $0.12 \mu \mathrm{M}$.

\section{Detection of Neurotransmitters}

In neuroimaging, there is a great demand to develop molecular imaging solutions to directly observe neural activity. Various biomarkers could be potentially exploited for direct monitoring of brain activation, the most obvious choices involve membrane potential changes or concentration changes of ions $\left(\mathrm{H}^{+}, \mathrm{Na}^{+}, \mathrm{K}^{+}, \mathrm{Cl}^{-}\right.$, $\mathrm{Ca}^{2+}, \mathrm{Mg}^{2+}$ ) or neurotransmitters. Current neuroscience relies on voltammetry and microdialysis for the detection and the quantification of neurotransmitter activity in the central nervous system. Both of them require highly invasive procedures and can only be realized in animal models. ${ }^{[42]}$ So far, little effort has been made to follow neurotransmitter concentrations with responsive MRI agents. Jasanoff et al. used protein engineering to obtain a paramagnetic heme-containing probe endowed with selectivity for dopamine instead of arachidonic acid.[43] This protein-based probe was successfully used after direct brain injection in rats to report on extracellular levels of dopamine following its release in reward-related brain stimulation. ${ }^{[44]}$ Parker et al. proposed $\mathrm{Gd}^{3+}$ complexes with binding functions specific of metabotropic glutamate- or N-methyl-D-aspartate (NMDA) receptors. ${ }^{[45]}$ Recently, we have reported the first responsive $\mathrm{Gd}^{3+}$-based MRI agents with a binding site for neurotransmitters. ${ }^{[46,47]}$ We wanted to propose a synthetic molecular platform which is smaller than engineered proteins. Our molecules include appropriate neurotransmitter recognition sites and they are capable of providing an MR intensity change upon neurotransmitter binding. Our objective was to target zwitterionic amino acid neurotransmitters; but selectivity to individual neurotransmitters was not an absolute necessity. The ultimate goal is to create re-

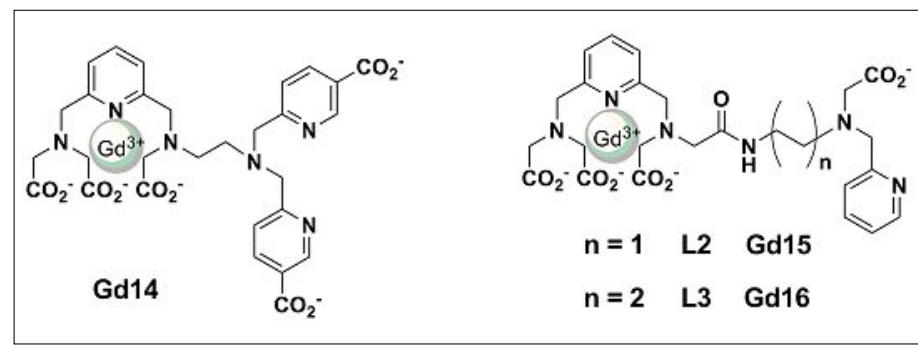

Fig. 7. Pyridine-based contrast agents for $\mathrm{Zn}^{2+}$ detection. 
sponsive agents capable of tracking brain activity by possibly detecting the cumulative effect of concentration variation in several neurotransmitters.

For the design of the neurotransmitterbinding receptor units, we considered that zwitterionic amino acids are bifunctional guests. Therefore, the receptors have to provide a specific binding site adapted for both $\mathrm{NH}_{3}^{+}$and $\mathrm{COO}^{-}$groups, which have to be situated within the molecule at an appropriate distance. This can allow for bivalent interactions which can ensure stronger and more selective binding than monovalent ones and lead to increased stability of the host-guest complex. ${ }^{[48]}$ To achieve this, our imaging probes provide ditopic interactions i) between a positively charged and coordinatively unsaturated $\mathrm{Gd}^{3+}$ chelate and the carboxylate group of the zwitterionic neurotransmitters and ii) between an azacrown ether conjugated to the $\mathrm{Gd}^{3+}$ chelate and the amine group of the neurotransmitters. The positive charge was achieved by using a GdDO2Abisamide derivative chelate. Crown ethers were selected for amine binding since they are among the best synthetic receptors for the recognition of ammonium ions and have been explored as essential elements in amino acid recognition. ${ }^{[49]}$ In these $\mathrm{Ln}^{3+}$ complexes, the relaxivity response originates from the substitution of the hydration water molecule by the neurotransmitter (Fig. 8). Consequently, neurotransmitter binding to these complexes led to a remarkable relaxivity decrease $(\sim 80 \%$, 'turn-off' response), which in turn should lead to a decreased signal intensity on the MR images. Several complexes have been synthesized within this family, bearing mono- or triaza crown ethers. All of them show selectivity towards zwitterionic over non-zwitterionic neurotransmitters, such as acetylcholine or serotonin. One of these complexes was successfully used in an $e x$ vivo acute mouse brain slice experiment to detect neural activity by MRI.[46] Intense neural activity was promoted by the injection of $\mathrm{KCl}(40 \mathrm{mM})$ into the artificial cerebrospinal fluid in which the brain slices were kept. Potassium ions induce membrane potential depolarization which is followed by neurotransmitter release. We found that following $\mathrm{KCl}$ stimulation, $\mathrm{MR}$ signal intensity was consistently decreasing in the cortex, the striatum and the hippocampus, while the medium did not undergo significant intensity change (Fig. 9). Interestingly, the signal intensity-decrease was the greatest in the hippocampus (dark spot), where the most significant neurotransmitter release is indeed expected. The intensity decrease observed was attributed to the cumulative effect of different zwitterionic neurotransmitters which, after release upon $\mathrm{KCl}$ stimulation, bind to

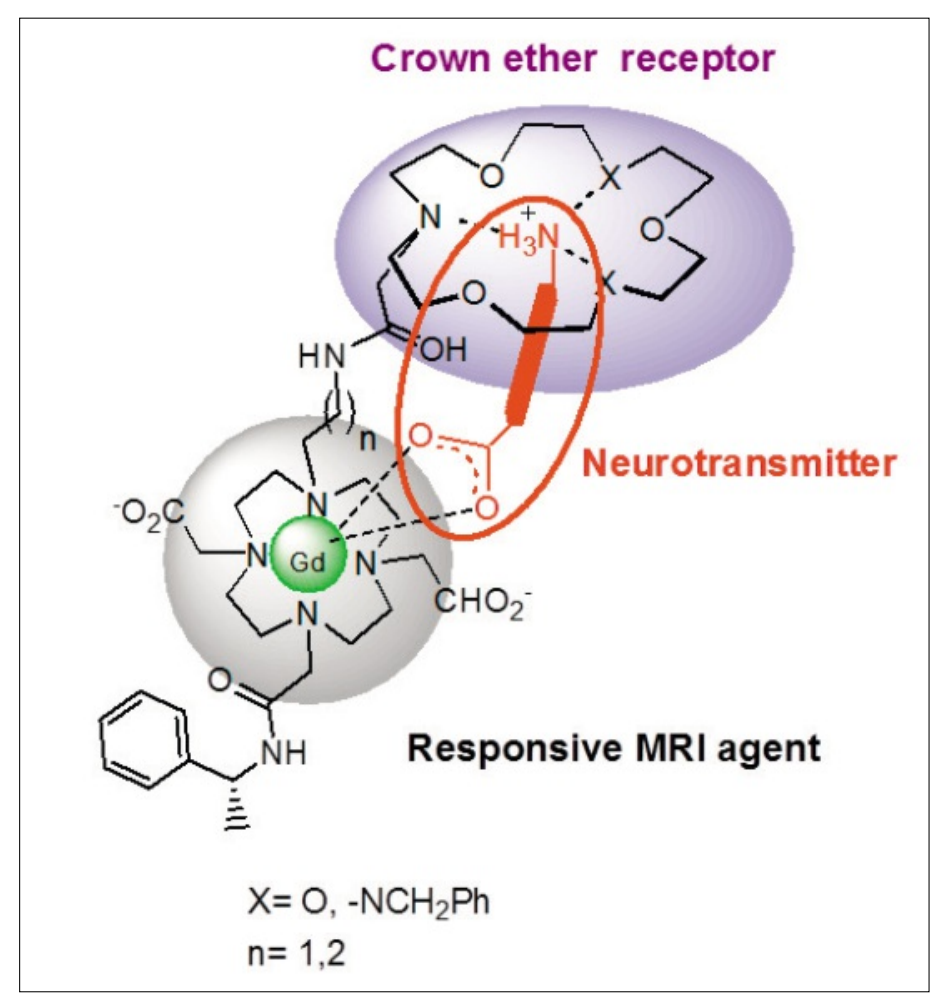

Fig. 8. The host-guest complex between the responsive probe and the zwitterionic amino acid neurotransmitters. Upon coordination to the $\mathrm{Gd}^{3+}$ ion, the carboxylate function replaces the water molecule coordinated to $\mathrm{Gd}^{3+}$, leading to a decrease of the proton relaxivity ('turn off' response).
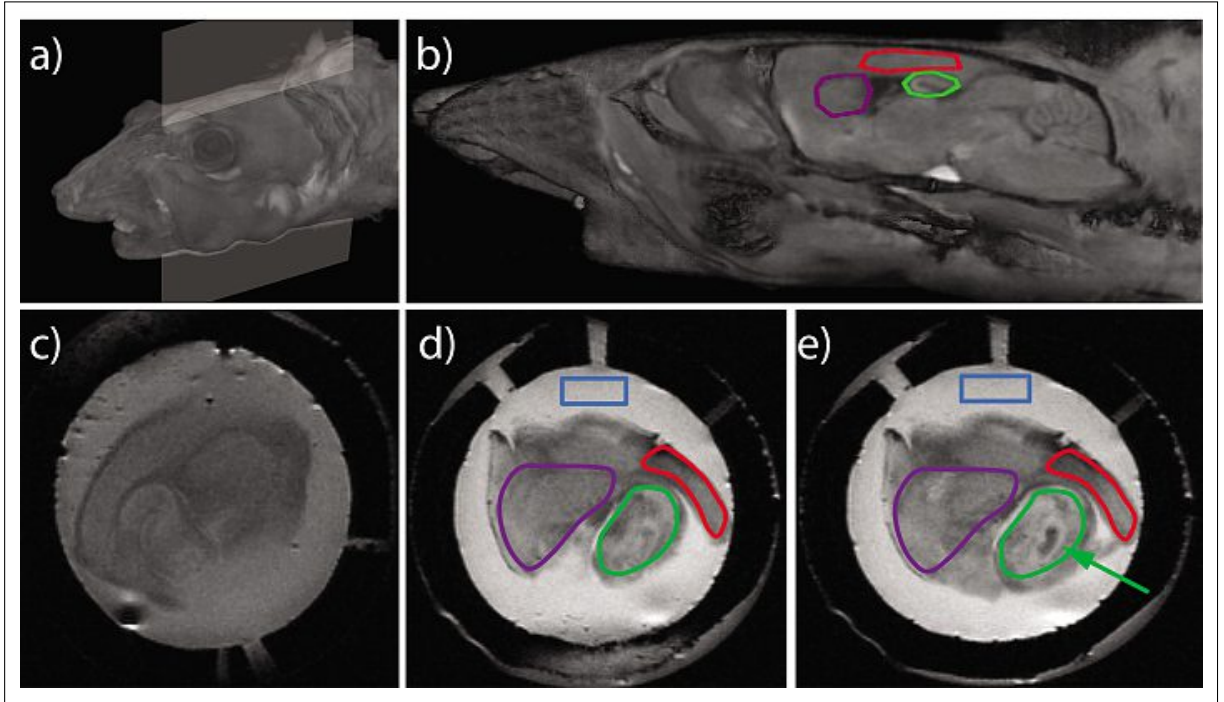

Fig. 9. Reconstituted 3D MR image of a mouse head showing the orientation of the examined slice (a) and the brain structures with the regions of interest (ROIs) (b). MR image of a mouse brain slice in the absence (c) and in the presence of $500 \mu \mathrm{M} \mathrm{GdL}$ with the ROls indicated (d): medium (blue), striatum (purple), cortex (red) and hippocampus (green). MR image of the same slice 10 minutes after $\mathrm{KCl}$ addition e). The highest signal intensity decrease is observed in the center of the hippocampus (indicated with the arrow). Reproduced with permission from ACS Chem. Neurosci. 2015, 6, 219-225.[46]

the $\mathrm{Gd}^{3+}$ complex and lead to its relaxivity decrease.

\section{Conclusion}

In the last decade, the research on responsive $\mathrm{Gd}^{3+}$-based or PARACEST MRI probes has exploded to target various biomarkers such as enzymes, biologically important cations or neurotransmitters, among many others. Some of these smart agents have successfully progressed into ex vivo or in vivo studies. Major current challenges involve a better specificity of the probes, a better response to decrease the detection limits, or to achieve a quantification of the biomarker by imaging. It is now widely accepted that novel breakthroughs in molecular imaging require an important input from chemistry, in particular coordination chemistry.

Revised: May 20, 2015 
[1] 'The Chemistry of Contrast Agents in Medical Magnetic Resonance Imaging', Second Edition, John Wiley \& Sons, Chichester, 2013.

[2] M. Woods, E. W. C. Donald, A. D. Sherry, Chem. Soc. Rev. 2006, 35, 500.

[3] P. Caravan, J. J. Ellison, T. J. McMurry, R. B. Lauffer, Chem. Rev. 1999, 99, 2293.

[4] C. S. Bonnet, L. Tei, M. Botta, E. Toth, in 'The Chemistry of Contrast Agents in Medical Magnetic Resonance Imaging', Second Edition, Eds. A. E. Merbach, L. Helm, E. Toth, John Wiley \& Sons, Chichester, 2013, pp. 343-385.

[5] R. A. Moats, S. E. Fraser, T. J. Meade, Angew. Chem. Int. Ed. 1997, 36, 726.

[6] a) C. S. Bonnet, E. Toth, Am. J. Neurorad. 2010, 31, 401; b) D. V. Hingorani, B. Yoo, A. S. Bernstein, M. D. Pagel, Chem.-Eur. J. 2014, 20, 9840; c) C. S. Bonnet, E. Toth, in 'Supramolecular Chemistry: From Molecules to Nanomaterials', Vol. 5, Eds. P. A. Gale, J. W. Steed, John Wiley \& Sons, Ltd., Chichester, 2012.

[7] A. Y. Louie, M. M. Huber, E. T. Ahrens, U. Rothbacher, R. Moats, R. E. Jacobs, S. E. Fraser, T. J. Meade, Nat. Biotech. 2000, 18, 321.

[8] J. A. Duimstra, F. J. Femia, T. J. Meade, J. Am. Chem. Soc. 2005, 127, 12847.

[9] a) M. Querol, D. G. Bennett, C. Sotak, H. W. Kang, A. Bogdanov, Chembiochem 2007, 8, 1637; b) M. Querol, J. W. Chen, R. Weissleder, A. Bogdanov, Org. Lett. 2005, 7, 1719.

[10] a) J. W. Chen, W. Pham, R. Weissleder, A. Bogdanov, Magn. Reson. Med. 2004, 52, 1021; b) M. O. Breckwoldt, J. W. Chen, L. Stangenberg, E. Aikawa, E. Rodriguez, S. M. Qiu, M. A. Moskowitz, R. Weissleder, Proc. Natl. Acad. Sci. USA 2008, 105, 18584.

[11] A. L. Nivorozhkin, A. F. Kolodziej, P. Caravan, M. T. Greenfield, R. B. Lauffer, T. J. McMurry, Angew. Chem. Int. Ed. 2001, 40, 2903.

[12] R. Napolitano, G. Pariani, F. Fedeli, Z. Baranyai, M. Aswendt, S. Aime, E. Gianolio, $J$. Med. Chem. 2013, 56, 2466.

[13] R. Lebel, B. Jastrzebska, H. Therriault, M. M. Cournoyer, J. O. McIntyre, E. Escher, W. Neugebauer, B. Paquette, M. Lepage, Magn. Reson. Med. 2008, 60, 1056.

[14] S. Mizukami, R. Takikawa, F. Sugihara, Y. Hori, H. Tochio, M. Wälchli, M. Shirakawa, K. Kikuchi, J. Am. Chem. Soc. 2007, 130, 794.

[15] B. Yoo, M. D. Pagel, J. Am. Chem. Soc. 2006, $128,14032$.

[16] B. Yoo, V. R. Sheth, M. D. Pagel, Tetrahedron Lett. 2009, 50, 4459.
[17] D. V. Hingorani, E. A. Randtke, M. D. Pagel, J. Am. Chem. Soc. 2013, 135, 6396.

[18] a) T. Chauvin, S. Torres, R. Rosseto, J. Kotek, B. Badet, P. Durand, É. Tóth, Chem.-Eur. J. 2012, 18, 1408; b) T. Chauvin, P. Durand, M. Bernier, H. Meudal, B. T. Doan, F. Noury, B. Badet, J. C. Beloeil, E. Toth, Angew. Chem. Int. Ed. 2008, 47, 4370.

[19] C. S. Bonnet, E. Toth, Future Med. Chem. 2010 , 2, 367 .

[20] W. H. Li, S. E. Fraser, T. J. Meade, J. Am. Chem. Soc. 1999, 121, 1413.

[21] R. Y. Tsien, Biochemistry 1980, 19, 2396.

[22] K. Dhingra, P. Fouskova, G. Angelovski, M. E. Maier, N. K. Logothetis, E. Toth, J. Bio. Inorg. Chem. 2008, 13, 35.

[23] A. Mishra, P. Fouskova, G. Angelovski, E. Balogh, A. K. Mishra, N. K. Logothetis, E. Toth, Inorg. Chem. 2008, 47, 1370.

[24] G. Angelovski, P. Fouskova, I. Mamedov, S. Canals, E. Toth, N. K. Logothetis, ChemBiochem 2008, 9, 1729.

[25] G. Angelovski, S. Gottschalk, M. Milosevic, J. Engelmann, G. E. Hagberg, P. Kadjane, P. Andjus, N. K. Logothetis, ACS Chem. Neurosci. 2014, 5, 360 .

[26] P. Kadjane, C. Platas-Iglesias, P. Boehm-Sturm, V. Truffault, G. E. Hagberg, M. Hoehn, N. K. Logothetis, G. Angelovski, Chem.-Eur. J. 2014, 20, 7351 .

[27] S. Guenduez, N. Nitta, S. Vibhute, S. Shibata, M. E. Mayer, N. K. Logothetis, I. Aoki, G. Angelovski, Chem. Commun. 2015, 51, 2782.

[28] G. Angelovski, T. Chauvin, R. Pohmann, N. K. Logothetis, E. Toth, Bioorg. Med. Chem. 2011, 19, 1097.

[29] C. J. Frederickson, J.-Y. Koh, A. I. Bush, Nat. Rev. Neurosci. 2005, 6, 449.

[30] a) A. Mishra, N. K. Logothetis, D. Parker, Chem.-Eur. J. 2011, 17, 1529; b) L. M. Matosziuk, J. H. Leibowitz, M. C. Heffern, K. W. MacRenaris, M. A. Ratner, T. J. Meade, Inorg. Chem. 2013, 52, 12250.

[31] a) K. Hanaoka, K. Kikuchi, Y. Urano, T. Nagano, J. Chem. Soc., Perkin Trans. 2 2001, 1840; b) K. Hanaoka, K. Kikuchi, Y. Urano, M. Narazaki, T. Yokawa, S. Sakamoto, K. Yamaguchi, T. Nagano, Chem. Biol. 2002, 9, 1027.

[32] J. L. Major, R. M. Boiteau, T. J. Meade, Inorg. Chem. 2008, 47, 10788.

[33] J. L. Major, G. Parigi, C. Luchinat, T. J. Meade, Proc. Natl. Acad. Sci. USA 2007, 104, 13881.

[34] A. C. Esqueda, J. A. Lopez, G. Andreu-DeRiquer, J. C. Alvarado-Monzon, J. Ratnakar,
A. J. M. Lubag, A. D. Sherry, L. M. De LeonRodriguez, J. Am. Chem. Soc. 2009, 131, 11387.

[35] A. J. M. Lubag, L. M. De Leon-Rodriguez, S. C. Burgess, A. D. Sherry, Proc. Natl. Acad. Sci. USA 2011, 108, 18400.

[36] C. S. Bonnet, F. Caille, A. Pallier, J.-F. Morfin, S. Petoud, F. Suzenet, E. Toth, Chem.-Eur. J. 2014, 20, 10959.

[37] C. S. Bonnet, F. Buron, F. Caille, C. M. Shade, B. Drahos, L. Pellegatti, J. Zhang, S. Villette, L. Helm, C. Pichon, F. Suzenet, S. Petoud, E. Toth, Chem.-Eur. J. 2012, 18, 1419.

[38] L. M. De Leon-Rodriguez, A. J. M. Lubag, J. A. Lopez, G. Andreu-de-Riquer, J. C. AlvaradoMonzon, A. D. Sherry, MedChemComm 2012, 3,480 .

[39] J. Luo, W. S. Li, P. Xu, L. Y. Zhang, Z. N. Chen, Inorg. Chem. 2012, 51, 9508.

[40] Y.-Q. Xu, J. Luo, Z.-N. Chen, Eur. J. Inorg. Chem. 2014, 3208

[41] R. Trokowski, J. M. Ren, F. K. Kalman, A. D. Sherry, Angew. Chem. Int. Ed. 2005, 44, 6920.

[42] a) C. S. Chaurasia, Biomed. Chromatogr: BMC 1999, 13, 317; b) P. Nandi, S. M. Lunte, Anal. Chim. Acta 2009, 651, 1; c) N. Dale, S. Hatz, F. Tian, E. Llaudet, Trends Biotechnol. 2005, 23, 420; d) M. L. Heien, M. A. Johnson, R. M. Wightman, Anal. Chem. 2004, 76, 5697.

[43] a) M. G. Shapiro, G. G. Westmeyer, P. A. Romero, J. O. Szablowski, B. Kuster, A. Shah, C. R. Otey, R. Langer, F. H. Arnold, A. Jasanoff, Nat. Biotech. 2010, 28, 264; b) E. M. Brustad, V. S. Lelyveld, C. D. Snow, N. Crook, S. T. Jung, F. M. Martinez, T. J. Scholl, A. Jasanoff, F. H. Arnold, J. Mol. Bio. 2012, 422, 245.

[44] T. Lee, L. X. Cai, V. S. Lelyveld, A. Hai, A. Jasanoff, Science 2014, 344, 533.

[45] a) A. Mishra, S. Gottschalk, J. Engelmann, D. Parker, Chem. Sci. 2012, 3, 131; b) N. Sim, S. Gottschalk, R. Pal, J. Engelmann, D. Parker, A. Mishra, Chem. Sci. 2013, 4, 3148.

[46] F. Oukhatar, S. Meme, W. Meme, F. Szeremeta, N. K. Logothetis, G. Angelovski, E. Toth, ACS Chem. Neurosci. 2015, 6, 219.

[47] F. Oukhatar, H. Meudal, C. Landon, N. K. Logothetis, C. Platas-Iglesias, G. Angelovski, E. Toth, Chem.-Eur. J. 2015, 21, 11226.

[48] a) M. Mammen, S. K. Choi, G. M. Whitesides, Angew. Chem. Int. Ed. 1998, 37, 2755; b) M.-K. Chung, S. J. Lee, M. L. Waters, M. R. Gagne, J. Am. Chem. Soc. 2012, 134, 11430.

[49] H. Tsukube, S. Shinoda, Chem. Rev. 2002, 102, 2389. 